\title{
Asynchronous DNA Replication of Biallelically Expressed Genes in Human Peripheral Blood Lymphocytes as a Prognostic Sign of Cancer
}

\author{
DOI: 10.17691/stm2021.13.3.04
}

Received August 25, 2020

V.V. Tsepenko, PhD, Senior Researcher, Laboratory of Molecular and Genetic Pathology,

Department of Clinical Morphology;

T.G. Shkavrova, PhD, Senior Researcher, Laboratory of Molecular and Genetic Pathology,

Department of Clinical Morphology;

V.N. Cherkesov, MD, PhD, Head of the Laboratory for Quality Control of Medical Care;

E.V. Golub, DSc, Leading Researcher, Laboratory of Molecular and Genetic Pathology,

Department of Clinical Morphology;

G.F. Mikhailova, DSc, Head of the Laboratory of Molecular and Genetic Pathology,

Department of Clinical Morphology

A. Tsyb Medical Radiological Research Centre - Branch of the National Medical Research Radiological Centre of the Ministry of Health of the Russian Federation, 4 Korolev St., Obninsk, 249036, Russia

The aim of the study was to identify and quantify lymphocytes with asynchronous replication of the AURKA and TP53 genes in cancer patients versus controls and to assess the diagnostic capabilities of this approach.

Materials and Methods. The study was carried out with peripheral blood lymphocytes probed for the AURKA and TP53 genes using the interphase fluorescence in situ hybridization (FISH) method (Vysis, USA and Kreatech, The Netherlands). The control group included 70 people: clinically healthy donors and patients with non-oncological diseases such as gastritis, pancreatitis, chronic calculous cholecystitis, bronchial asthma, peptic ulcer disease, inguinal hernia, arthrosis, myoma, hepatitis, epilepsy, chronic prostatitis, chronic tonsillitis, and rectal adenoma. The group of cancer patients included 219 people with various oncological diseases: gastric cancer ( $n=68)$, colorectal cancer $(n=30)$, chronic lymphocytic leukemia $(n=52)$, Hodgkin lymphoma $(n=33)$, and polyneoplasia $(n=41)$.

Results. In the control group, the mean frequency of lymphocytes with asynchronous gene replication (AGR) was $22.0 \pm 3.4 \%$ for AURKA and $18.0 \pm 3.2 \%$ for TP53; in the group of cancer patients, that was $36.8 \pm 4.8$ and $28.4 \pm 5.1 \%$, respectively. The excessive presence of lymphocytes with the AGR in cancer patients was consistent and statistically significant $(p<0.0001)$. For the AURKA gene, the AGRbased cancer detection showed a sensitivity of $98.6 \pm 0.7 \%$, a specificity of $100 \%$, and an accuracy of $98.3 \pm 0.8 \%$, and for the TP53 gene $78.6 \pm 3.1,98.5 \pm 0.9$, and $85.9 \pm 2.6 \%$, respectively.

Conclusion. This pilot study on lymphocytes with AGR of AURKA and TP53 genes in cancer patients can serve a basis for creating a new molecular cytogenetic technology for detecting malignant neoplasms in humans.

Key words: asynchronous DNA replication; fluorescence in situ hybridization; FISH; peripheral blood lymphocytes; AURKA; TP53; diagnosis of malignant neoplasms.

How to cite: Tsepenko V.V., Shkavrova T.G., Cherkesov V.N., Golub E.V., Mikhailova G.F. Asynchronous DNA replication of biallelically expressed genes in human peripheral blood lymphocytes as a prognostic sign of cancer. Sovremennye tehnologii v medicine 2021; 13(3): 33, https://doi.org/10.17691/stm2021.13.3.04

This is an open access article under the CC BY 4.0 license (https://creativecommons.org/licenses/by/4.0/).

Corresponding author: Victoria V. Tsepenko, e-mail: mgp@mrrc.obninsk.ru 


\section{Introduction}

Early diagnosis of malignant neoplasms is one of the most important problems of clinical medicine. In the Russian Federation, in the single year of 2018, cancer was diagnosed for the first time in 624,709 people. With the diagnosis made at stages I-II, the patient can make a recovery with a probability of about $80 \%$. However, in Russia, only about $55 \%$ of diagnoses are made at stages I or II [1]. In many cases, cancer is detected either when people seek medical assistance for their symptoms, or during massive screening examinations aimed at detecting this specific disease. In this regard, the methodology of cancer screening is of great importance. This should be highly sensitive, specific, and near $100 \%$ accurate; in clinical practice, however, very few methods meet these requirements.

By now, the screening programs for breast, cervical, and colorectal cancers have been proved most effective. Among the detection methods, low-dose spiral computed tomography is increasingly popular. It is characterized by high sensitivity that significantly increases the likelihood of detecting even small tumors, but the method has low specificity. In addition, the patient's body is exposed to ionizing radiation, which in itself is a risk factor for developing cancer. Of the instrumental methods for early diagnosis of cancer, ultrasound imaging should also be noted: this is the most accessible and simple modality that has no contraindications. Up to date, a lot of practical experience in using ultrasound for diagnosing cancer of the abdominal cavity and the thyroid has been accumulated.

Identifying an oncological disease at the preclinical stage is rather difficult. Therefore, in recent decades, methods of molecular genetics and cytogenetics have been increasingly used to diagnose early-stage cancers. These methods make it possible to detect abnormalities in gene expression, DNA methylation, mutations, and structural aberrations of DNA, as well as specific features of the replication processes characteristic of oncological diseases. As reported, deviations in replication timing indicate that asynchronous replication of biallelically expressed genes often correlates with the development of cancer [2-6].

In an early study [7], it was shown that the level of blood lymphocytes with asynchronous gene replication (AGR) in healthy donors did not exceed $14 \%$ for either the TP53 gene or locus $21 \mathrm{q} 22$, while in patients with chronic lymphocytic leukemia, this level was twice as high for both the gene and the locus. Later, similar results were obtained in patients with solid tumors. In [8], the authors showed that in patients with renal cell carcinomas, the number of lymphocytes with AGR for the TP53 gene and locus 21q22 was 36 and 44\%, respectively, while in healthy individuals, the numbers were much lower: 12 and 14\%. Subsequently, such studies were carried out with other genes both in healthy controls and in cancer patients [9].
Here, for the first time, we present the data on asynchronous replication of the AURKA gene in peripheral blood lymphocytes from clinically healthy individuals, patients with non-oncological diseases, and patients with several types of cancer.

The aim of the study was to identify and quantify lymphocytes with asynchronous replication of the AURKA and TP53 genes in cancer patients versus controls and to assess the diagnostic capabilities of this approach.

\section{Materials and Methods}

Studied groups. The study involved a control group and a group of patients diagnosed with various cancers. The control group included 40 clinically healthy individuals and 30 patients with a history of non-oncological diseases: gastritis (6), pancreatitis (2), chronic calculous cholecystitis (11), bronchial asthma (1), peptic ulcer (1), inguinal hernia (4), arthrosis (1), myoma (1), hepatitis (1), epilepsy (1), chronic prostatitis (2), chronic tonsillitis (3), and rectal adenoma (1). Some of the patients had more than one diagnosis. In total, 34 men and 36 women aged from 21 to 77 years old (average 42) were examined.

The group of patients with cancer included 219 individuals (123 men and 96 women) aged from 21 to 88 years old (average 61). The diagnoses were distributed as follows: gastric cancer (68), colorectal cancer (30), chronic lymphocytic leukemia (52), Hodgkin lymphoma (33), and polyneoplasia (41). The polyneoplasia group included patients with two or more synchronous or metachronous tumors: melanoma, cancers of the colon, sigmoid, or ileum, gastric cancer, larynx, esophagus, kidney, lung, ovaries, breast, cervix, prostate, bladder, and thyroid cancer. The cancer stages in the examined patients varied from IA to IV.

Before taking samples, all healthy donors and noncancer patients were informed in detail about the study; the informed consent form was signed by each participant. Studies in cancer patients were carried out according to the clinical protocols approved by the Ministry of Health of the Russian Federation and received an approval from the Ethics Committee of the A. Tsyb Medical Radiological Research Centre (Russia).

\section{Studied genes}

The TP53 suppressor gene. The tumor suppressor gene TP53, located at locus 13.1 on the p-arm of chromosome 17, plays an important role in apoptosis, DNA repair, and cell cycle regulation in the late $\mathrm{G} 1$ phase. Inactivation of TP53 is a key mechanism in malignant cell transformation.

Abnormalities of the TP53 gene are commonly found in various human cancers. The significance of TP53 for tumor suppression has been confirmed by demonstrating a high rate of tumorigenesis in TP53-deficient mice and a high risk of early cancers in humans with hereditary mutations in the TP53 gene (Li-Fraumeni syndrome). Inactivation of this gene was noted in half of all sporadic 
Figure 1. Asynchronous replication of the TP53 gene in human peripheral blood lymphocytes:

(a) normal lymphocyte (two single signals); (b) lymphocyte with impaired synchronicity of gene replication (one single and one double signal); $\times 1000$
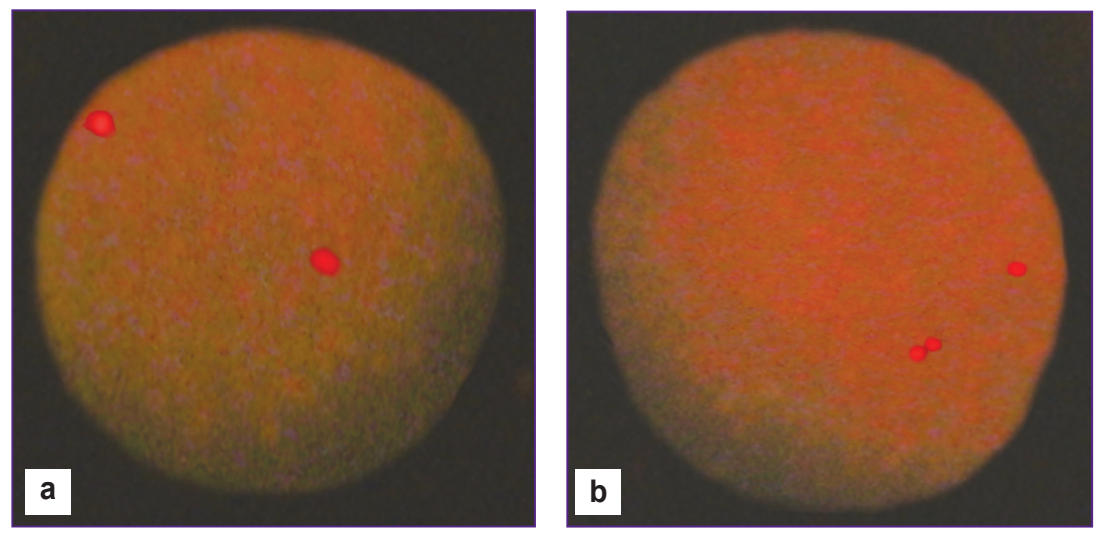

cancers. Moreover, in cancers in which the TP53 gene remains intact, its function is often impaired. TP53 plays a fundamental role in controlling the cell proliferation and maintaining the integrity and stability of the genome. As reported, human malignant neoplasms are associated with genetic or epigenetic mechanisms that counteract the TP53-dependent signaling pathway [10-12].

The AURKA protooncogene. The AURKA gene located at locus 13.2 of the q-arm of chromosome 20, encodes for the Aurora kinase family. It is a family of serine/threonine kinases essential for normal mitosis and/or meiosis in eukaryotic cells. Aurora kinases play a crucial role in the regulation of mitotic events such as the assembly of the division spindle, cytokinesis, and the centrosome/cytoskeleton function. The AURKA gene is expressed in the centrosome during prophase, ensuring its division and maturation. During metaphase, this gene is located in polar microtubules and ensures the assembly of the division spindle; during cytokinesis, it is located in the Fleming's body.

The AURKA gene is an oncogene. In animals, an increased activity of this gene causes genetic instability and, subsequently, tumor formation [13]. In addition, the gene is located in a region, which is often amplified in human cancers, and its mutations increase the risk of developing esophageal, ovarian, lung, and breast cancer. Abnormalities in AURKA expression could lead to disorders of the mitotic cycle resulting in gastric, hepatocellular, and pancreatic cancers [14-16].

Interphase fluorescence in situ hybridization (FISH) on lymphocytes of peripheral blood. Samples of venous blood (4-6 ml) were taken using a vacuum system containing Li-heparin at a concentration of 12 $30 \mathrm{IU}$ per $1 \mathrm{ml}$ of blood. These whole blood samples were diluted $(1: 9)$ with a warm $\left(37^{\circ} \mathrm{C}\right)$ solution of $\mathrm{KCl}$ $(550 \mathrm{mg}+110 \mathrm{ml})$ and placed in a thermostat $\left(37^{\circ} \mathrm{C}\right)$ for $30 \mathrm{~min}$. Then, the cells were fixated in a mixture of methanol: acetic acid (3:1). Aliquots of the cell suspension $(20-30 \mu \mathrm{l})$ were applied onto recently frozen glass slides. We used commercially available DNA probes from Vysis (USA) and Kreatech (The Netherlands) for the AURKA and TP53 genes. Pre- and post-hybridization washes were performed according to the manufacturer's instructions. A Thermobrite hybridizer (StatSpin, USA) was used for denaturation and hybridization.

Statistical data analysis. The slides were scored independently by two researchers using an Axio Imager A-2 fluorescence microscope (Carl Zeiss, Germany) equipped with the DAPI, Orange/Green, and Gold filters (Vysis). For each blood sample, 300-900 interphase cells with clear fluorescent signals were analyzed (Figure 1).

Statistical analysis was performed using the SPSS Statistics v. 23 and Microsoft Office Excel (2007). The resulting data were combined into variational series, for which the mean value, standard deviation, and $95 \%$ confidence interval $(\mathrm{Cl})$ were calculated. The data distribution was tested for normality using the onesample Kolmogorov-Smirnov test. The sets were found homogeneous. The data were presented as mean \pm standard deviation $(M \pm \sigma)$. The significance of the differences between the mean values was assessed using one-way analysis of variance and two-tailed Student's t-test with Newman-Keuls correction for multiple comparisons. Differences were considered statistically significant at $p<0.05$. To assess the informative value and the resolution power of the test, we calculated its sensitivity, specificity, and accuracy. The specificity of the method was determined from the proportion of cancer-free subjects among the "negative" analyzes; the sensitivity - as the proportion of subjects with confirmed cancer among the "positive" tests. The accuracy was calculated as the proportion of "exact matches" between the test results and the diagnoses among all subjects.

\section{Results}

The mean frequency for lymphocytes with asynchronous replication of AURKA and TP53 was higher among cancer patients than among non-cancer controls (Table 1).

For the AURKA gene, this value was significantly higher in all subgroups of cancer patients as compared with that in non-cancer patients or healthy subjects $(p<0.05)$. 


\section{BIOTECHNOLOGIES}

Table 1

Occurrence of lymphocytes with asynchronous replication of AURKA and TP53 in the examined groups

\begin{tabular}{|c|c|c|c|c|}
\hline Groups & $\begin{array}{l}\text { Number } \\
\text { of subjects } \\
\text { examined }\end{array}$ & $\begin{array}{c}\text { Number } \\
\text { of cells analyzed }\end{array}$ & $\begin{array}{l}\text { Lymphocytes } \\
\text { with AGR, } \\
\text { min-max (\%) }\end{array}$ & $\begin{array}{l}M \pm \sigma \\
(\%)\end{array}$ \\
\hline \multicolumn{5}{|c|}{ AURKA gene } \\
\hline Control & 70 & 22,084 & $13.7-27.7$ & $22.0 \pm 3.4$ \\
\hline \multicolumn{5}{|l|}{ Cancer patients: } \\
\hline gastric cancer & 65 & 20,083 & $28.6-42.0$ & $33.9 \pm 2.9$ \\
\hline colorectal cancer & 30 & 9,000 & $32.0-44.3$ & $35.5 \pm 2.9$ \\
\hline polyneoplasia & 41 & 12,404 & $31.3-46.0$ & $39.6 \pm 3.8$ \\
\hline solid tumors (total) & 136 & 41,487 & $28.6-46.0$ & $36.3 \pm 6.8$ \\
\hline chronic lymphocytic leukemia & 52 & 15,600 & $28.0-55.3$ & $38.4 \pm 6.3$ \\
\hline Hodgkin lymphoma & 31 & 9,300 & $30.0-51.3$ & $38.0 \pm 4.4$ \\
\hline hematology (total) & 83 & 24,900 & $28.0-55.3$ & $38.2 \pm 5.8$ \\
\hline \multicolumn{5}{|c|}{ TP53 gene } \\
\hline Control & 65 & 20,576 & $11.7-25.3$ & $18.0 \pm 3.2$ \\
\hline \multicolumn{5}{|l|}{ Cancer patients: } \\
\hline gastric cancer + colorectal cancer & 71 & 20,967 & $18.3-38.9$ & $26.1 \pm 4.2$ \\
\hline polyneoplasia & 41 & 11,995 & $21.0-44.4$ & $32.5 \pm 3.9$ \\
\hline solid tumors (total) & 112 & 32,962 & $18.3-44.4$ & $28.4 \pm 5.1$ \\
\hline
\end{tabular}

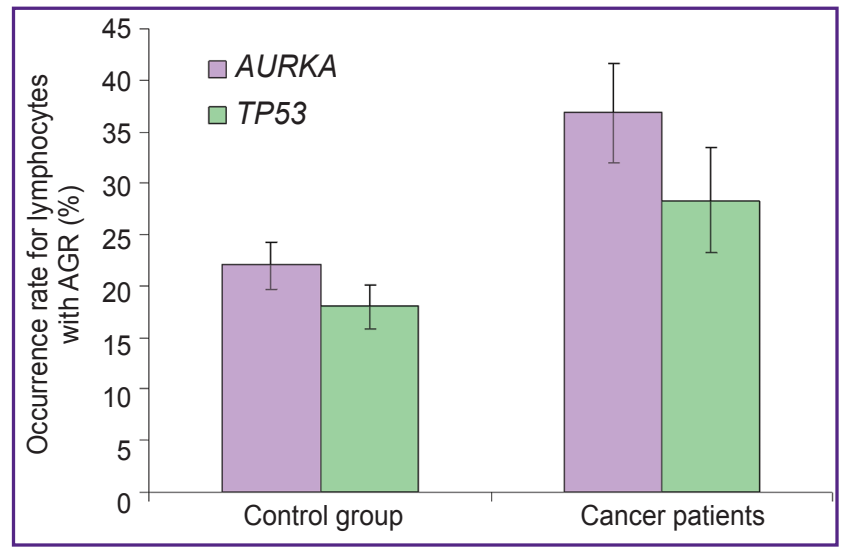

Figure 2. The mean frequency for lymphocytes with asynchronous replication of the AURKA and TP53 genes in the control group and in cancer patients. Two-sided Student's t-test; $M \pm \sigma ; p<0.05$

Among the cancer subgroups, the differences between patients with gastric cancer vs patients with polyneoplasia were statistically significant $(p=0.023)$. There were no significant differences between the subgroups of gastric vs colorectal cancer or between chronic lymphocytic leukemia vs Hodgkin lymphoma $(p=0.57$ and $p=0.91$, respectively). After all cancer patients were combined and re-divided into subgroups of patients with either solid tumors or with oncohematology, there were still no significant differences between these two subgroups $(p=0.274)$. Based on that, we then united these two subgroups into a single group of cancer patients.
For the TP53 gene, the frequency of AGR in the polyneoplasia subgroup and the "gastric cancer + colorectal cancer" subgroup were significantly higher than those in control $(p=0.0001$ and $p=0.01)$. There was also a statistically significant difference in the presence of lymphocytes with AGR between the patients with gastric cancer and those with multiple tumors $(p=0.043)$.

As illustrated in Figure 2, the frequency for lymphocytes with asynchronous replication of the AURKA and TP53 genes in the combined group of cancer patients $(36.8 \pm 4.8$ and $28.4 \pm 5.1 \%$, respectively) significantly exceed the control levels $(22.0 \pm 3.4$ and $18.0 \pm 3.2 \%$, respectively) $(p=0.0001)$. The number of lymphocytes with AGR for AURKA significantly exceeded that for TP53 both in the group of cancer patients $(p=0.001)$ and in control $(p=0.03)$.

As a cut-off point separating the control from the group of cancer patients, the upper limit of $95 \% \mathrm{Cl}$ of the control group was taken. For the AURKA gene in the control group, this value was $28.7 \%$. In this group, there was no single person in whom the presence of lymphocytes with AGR for AURKA would exceed this level. In cancer patients though, the upper limit of $95 \%$ $\mathrm{Cl}$ exceeded the $28.7 \%$ value in 214 people, and in 5 people it was below this cut-off threshold.

For the TP53 gene, the upper limit of $95 \% \mathrm{Cl}$ in the control group was $24.2 \%$. Only 1 person showed the value exceeding this $24.2 \%$, and in 64 subjects of the control group, the occurrence rate of lymphocytes with AGR was within the $95 \% \mathrm{Cl}$. Among the cancer patients, 88 individuals showed this indicator exceeding the cutoff level and in 24 patients it was below the cut-off point. 
Table 2

Sensitivity, specificity, and accuracy of the method (\%) $(\mathrm{M} \pm \sigma)$

\begin{tabular}{lcc}
\hline \multirow{2}{*}{ Parameter } & \multicolumn{2}{c}{ Gene tested } \\
\cline { 2 - 3 } Sensitivity & AURKA & TP53 \\
\hline Specificity & $98.6 \pm 0.7$ & $78.6 \pm 3.1$ \\
\hline Accuracy & 100 & $98.5 \pm 0.9$ \\
\hline
\end{tabular}

The information value and the resolution capacity of using the asynchronous replication of AURKA and TP53 for making a diagnosis, are shown in Table 2.

\section{Discussion}

A major characteristic of cell malignant transformation is its uncontrolled growth often caused by increased mutagenesis and genome instability [17]. Therefore, the correct order of DNA replication is essential for normal cell division to ensure that the genetic information is passed on unchanged into the next cell generation. DNA replication is a strictly regulated process; in this, most of the homologous loci in the genome replicate synchronously but a small part of them replicate asynchronously [3, 18]. Disturbances in replication timing may affect gene expression, distort epigenetic modifications, and interfere with structural rearrangements. These events can lead to genome destabilization and, ultimately, to the development of cancer [5, 19]. As shown [6], two-thirds of mutations associated with cancer result from impaired DNA replication. Analysis of genome instability, cancer and impaired replication program [2] suggests that aberrant replication is an early event in carcinogenesis.

Using the method of interphase fluorescence in situ hybridization, a number of research groups studied peripheral blood lymphocytes with asynchronous replication of the TP53, HER-2/neu, C-MYC, RB1, and $A M L 1$ genes in humans and obtained the following results:

1) in clinically healthy individuals, the frequency of lymphocytes with AGR varies within 10-21\% [7, 8, 20-24];

2 ) in cancer patients (lymphomas [24, 25], chronic lymphocytic leukemia [7], chronic myeloid leukemia [25], renal cell carcinomas [8], prostate cancer [26], and breast cancer [21]), this indicator varies within $28-41 \%$;

3 ) both in oncohematological patients and in patients with solid tumors, AGR is observed in peripheral blood lymphocytes [7, 8];

4) the presence of lymphocytes with AGR increases with the grade of malignancy [23, 24];

5 ) the loss of synchrony of gene replication in cancer patients is a reversible epigenetic phenomenon associated, among other things, with abnormal methylation [20, 22].
Our study on lymphocytes with asynchronous replication of the TP53 gene both in cancer-free subjects and in patients with solid tumors showed good agreement with the main findings of others [8, 24, 27]. Thus, our results on the replication timing of biallelically expressed genes in blood lymphocytes from cancer patients $[9,28]$, suggest that $A G R$ can serve as a nonspecific marker of cancer.

It is known that malignant neoplasm in humans is a result of either oncogene activation or disruption of tumor suppressor genes. In this study, we selected the AURKA gene, which is an oncogene, and the TP53 gene, which is a tumor suppressor gene. The results show that the frequency of lymphocytes with AGR for AURKA is significantly higher than that for TP53 both in the control group and in cancer patients. Assuming that the occurrence of lymphocytes with asynchronous replication of biallelically expressed genes is a nonspecific tumor marker, we propose this indicator as a potential molecular cytogenetic test for identifying people with cancer. Table 2 shows the sensitivity, specificity, and accuracy of this test. According to the results, the most informative parameter would be the occurrence rate of lymphocytes with asynchronous replication of the AURKA gene since all three indices in this test exceed $98 \%$.

\section{Conclusion}

The level of peripheral blood lymphocytes with asynchronous replication of the AURKA gene can serve as a basic parameter for the development of a new molecular cytogenetic technology for detecting malignant neoplasms in humans. Creating such a technology would require further in-depth studies, in particular, the role of gender, age, smoking habits, and other factors reflecting the specific features of various cancers.

Study funding. There were no additional sources of funding for the study.

Conflicts of interest. The authors have no conflicts of interest related to this publication.

\section{References}

1. Zlokachestvennye novoobrazovaniya v Rossii v 2018 godu (zabolevaemost' $i$ smertnost') [Malignant neoplasms in Russia in 2018 (morbidity and mortality)]. Kaprin A.D., Starinskiy V.V., Petrova G.V. (editors). Moscow: MNIOI im. A. Gertsena - filial FGBU "NMITs radiologii" Minzdrava Rossii; 2019; 250 p.

2. Blumenfeld B., Ben-Zimra M., Simon I. Perturbations in the replication program contribute to genomic instability in cancer. Int J Mol Sci 2017; 18(6): 1138, https://doi.org/10.3390/ ijms18061138.

3. Hiratani I., Gilbert D.M. Replication timing as an epigenetic mark. Epigenetics 2009; 4(2): 93-97, https://doi. org/10.4161/epi.4.2.7772. 
4. Donley N., Thayer M.J. DNA replication timing, genome stability and cancer: late and/or delayed DNA replication timing is associated with increased genomic instability. Semin Cancer Biol 2013; 23(2): 80-89, https://doi.org/10.1016/j. semcancer.2013.01.001.

5. Ryba T., Battaglia D., Chang B.H., Shirley J.W., Buckley Q., Pope B.D., Devidas M., Druker B.J., Gilbert D.M. Abnormal developmental control of replication-timing domains in pediatric acute lymphoblastic leukemia. Genome Res 2012; 22(10): 1833-1844, https://doi.org/10.1101/gr.138511.112.

6. Tomasetti C., Li L., Vogelstein B. Stem cell divisions, somatic mutations, cancer etiology, and cancer prevention. Science 2017; 355(6331): 1330-1334, https://doi.org/10.1126/ science.aaf9011

7. Amiel A., Litmanovich T., Gaber E., Lishner M., Avivi L., Fejgin M.D. Asynchronous replication of p53 and 21q22 loci in chronic lymphocytic leukemia. Hum Genet 1997; 101(2): 219222, https://doi.org/10.1007/s004390050619.

8. Dotan Z.A., Dotan A., Litmanovitch T., Ravia Y., Oniashvili N., Leibovitch I., Ramon J., Avivi L. Modification in the inherent mode of allelic replication in lymphocytes of patients suffering from renal cell carcinoma: a novel genetic alteration associated with malignancy. Genes Chromosomes Cancer 2000; 27(3): 270-277, https://doi.org/10.1002/ (sici)1098-2264(200003)27:3<270::aid-gcc7>3.0.co;2-7.

9. Mikhailova G.F., Tsepenko V.V., Shkavrova T.G., Goloub E.V. Asynchronous replication in oncological patients. Uspehi molekularnoj onkologii 2018; 5(1): 26-34, https://doi.org/ 10.17650/2313-805x-2018-5-1-26-34.

10. Silwal-Pandit L., Langerød A., Børresen-Dale A.L. TP53 mutations in breast and ovarian cancer. Cold Spring Harb Perspect Med 2017; 7(1): a026252, https://doi.org/10.1101/ cshperspect.a026252.

11. Aubrey B.J., Strasser A., Kelly G.L. Tumor-suppressor functions of the TP53 pathway. Cold Spring Harb Perspect Med 2016; 6(5): a026062, https://doi.org/10.1101/cshperspect. a026062.

12. Torén W., Ansari D., Andersson R. Immunohistochemical investigation of prognostic biomarkers in resected colorectal liver metastases: a systematic review and meta-analysis. Cancer Cell Int 2018; 18(1): 217, https://doi.org/10.1186/s12935-018-0715-8.

13. Damodaran A.P., Vaufrey L., Gavard O., Gavard O., Prigent $\mathrm{C}$. Aurora A kinase is a priority pharmaceutical target for the treatment of cancers. Trends Pharmacol Sci 2017; 38(8): 687-700, https://doi.org/10.1016/j.tips.2017.05.003.

14. Seeling J.M., Farmer A.A., Mansfield A., Cho H., Choudhary $M$. Differential selective pressures experienced by the Aurora kinase gene family. Int J Mol Sci 2018; 19(1): 72, https:/doi.org/10.3390/jims 19010072.

15. Zhan S.J., Liu B., Linghu H. Identifying genes as potential prognostic indicators in patients with serous ovarian cancer resistant to carboplatin using integrated bioinformatics analysis. Oncol $\operatorname{Rep} 2018 ; \quad 39(6): 2653-2663$, https://doi. org/10.3892/or.2018.6383.

16. Zhou L., Du Y., Kong L., Zhang X., Chen Q. Identification of molecular target genes and key pathways in hepatocellular carcinoma by bioinformatics analysis. Onco Targets Ther 2018 11: 1861-1869, https://doi.org/10.2147/ott.s156737.

17. Hanahan D., Weinberg R.A. Hallmarks of cancer: the next generation. Cell 2011; 144(5): 646-674, https://doi. org/10.1016/j.cell.2011.02.013.

18. Klein K.N., Gilbert D.M. Epigenetic vs. sequencedependent control of eukaryotic replication timing. In: The initiation of DNA replication in eukaryotes. Kaplan D.L. (editor). Springer International Publishing Switzerland; 2016; p. 39-63, https://doi.org/10.1007/978-3-319-24696-3_3.

19. Macheret M., Halazonetis T.D. DNA replication stress as a hallmark of cancer. Annu Rev Pathol 2015; 10: 425-448, https://doi.org/10.1146/annurev-pathol-012414-040424.

20. Nagler A., Cytron S., Mashevich M., KorensteinIlan A., Avivi L. The aberrant asynchronous replication characterizing lymphocytes of cancer patients - is erased following stem cell transplantation. BMC Cancer 2010; 10: 230, https://doi.org/10.1186/1471-2407-10-230.

21. Grinberg-Rashi H., Cytron S., Gelman-Kohan Z., Litmanovitch T., Avivi L. Replication timing aberrations and aneuploidy in peripheral blood lymphocytes of breast cancer patients. Neoplasia 2010; 12(8): 668-674, https://doi.org/ 10.1593/neo.10568.

22. Korenstein-Ilan A., Amiel A., Lalezari S., Lishner M., Avivi L. Allele-specific replication associated with aneuploidy in blood cells of patients with hematologic malignancies. Cancer Genet Cytogenet 2002; 139(2): 97-103, https://doi. org/10.1016/s0165-4608(02)00610-6.

23. Amiel A., Kirgner I., Gaber E., Manor Y., Fejgin M., Lishner M. Replication pattern in cancer: asynchronous replication in multiple myeloma and in monoclonal gammopathy. Cancer Genet Cytogenet 1999; 108(1): 32-37, https://doi.org/10.1016/s0165-4608(98)00107-1.

24. Amiel A., Kitay-Cohen Y., Fejgin M.D., Lishner M. Replication status as a marker for predisposition for lymphoma in patients with chronic hepatitis $C$ with and without cryoglobulinemia. Exp Hematol 2000; 28(2): 156-160, https:// doi.org/10.1016/s0301-472x(99)00140-x.

25. Amiel A., Litmanovitch T., Lishner M., Mor A., Gaber E., Tangi I., Fejgin M., Avivi L. Temporal differences in replication timing of homologous loci in malignant cells derived from $\mathrm{CML}$ and lymphoma patients. Genes Chromosomes Cancer 1998; 22(3): 225-231, https://doi.org/10.1002/(sici)1098-2264 (199807)22:3<225::aid-gcc8>3.0.co;2-y.

26. Cytron S., Stepnov E., Bounkin I., Mashevich M., Dotan A., Avivi L. Epigenetic analyses in blood cells of men suspected of prostate cancer predict the outcome of biopsy better than serum PSA levels. Clin Epigenetics 2011; 2(2): 383-388, https://doi.org/10.1007/s13148-011-0029-3.

27. Dotan Z.A., Dotan A., Ramon J., Avivi L. Altered mode of allelic replication accompanied by aneuploidy in peripheral blood lymphocytes of prostate cancer patients. Int $\mathrm{J}$ Cancer 2004; 111(1): 60-66, https://doi.org/10.1002/ijc.20237.

28. Mikhaylova G.F., Tsepenko V.V., Shkavrova T.G., Golub E.V., Kaprin A.D. Method for screening malignant neoplasms in humans. Patent RU 2665965C1. 2017. 